\title{
MANIFESTATION OF INFORMATION PROPERTIES OF WATER SUFFERED CAVITATION IN AFTEREFFECT VIA EXAMPLES "MEMORIES OF SOILS"
}

\author{
Evgeniy Ivanov, Nikita Kokorin \\ Nizhny Novgorod State Agricultural Academy, Russia \\ ivanov.e.g@mail.ru,nikia_6500@mail.ru
}

\begin{abstract}
When sound waves of any intensity pass in water the water is broken in the vacuum phase and cavities appear. In the manometrical phase there is collapse of these cavities. The frequency is $f=1-3 \mathrm{kHz}$. If each cavity is collapsed in the travelling speed of $C=3000 \mathrm{~m} \cdot \mathrm{s}^{-1}$, a high energy density is created in the impact point. Such energy value provokes a multiple change of its forms, the increase in the temperature and pressure, creation of secondary shock waves, their interference, deformation of water in the level of molecule group, the change in the structure of clusters and their pollutants. The properties of water change depend on the cavitation treatment mode. It was established by biotesting. These specific qualities are preserved for a long time. Besides, a substance moistened by such water has such qualities which preserve even after the total drying of the water. When the substance was moistened for the second time by usual water the water received the properties of cavitated water. So, the water suffered cavitation transfer of the new properties to usual water through hard substances. It was established by the experiments that porous materials, smooth, as well as soil and grounds may be used as intermediator substance. These facts take place in polluted waters, in particularly, in salt water. It is of grand importance in agricultural areas with lack of fresh water. The established phenomenon permits to economize fresh water for irrigation. In this case at first we receive a little of "good" water manufactured of "bad" water by the cavitation method and use it for initial irrigation. Then we do irrigation with "bad" water, which takes on the properties of the "good" water.
\end{abstract}

Keywords: cavitation, water, quality, pollution, memory.

\section{Introduction}

Water is of grand importance for vital activity of people. Firstly, the functions of all the body systems decease if water does not come to the organism. Secondly, 80-90\% of human brain weight is water (the rest $10-20 \%$ is a reservoir for water). It is a common knowledge that brain provides accepting, processing, saving, and releasing of a grand volume of information. The information may be the result of any mental activity and besides it may be associated with management of vital processes on unconscious level.

Some information qualities of water activated by use of acoustic cavitation processes were established earlier. So, the method of stimulation of seed germination ability and of plant development as well as of cutting established [1] by ultrasound treatment is already known for some decennia. It is established by the authors of the article that the influence of ultrasound influence durability on the germination ability may be characterized by its periodic nature. An excessive intensity of treatment provokes the decrease in the germination ability.

The biologists S.S. Tarasov and A.P. Veselov established that each crop has its own susceptibility to ultrasound treatment and that the ultrasound treatment is the most rational in the seed swelling period [2;3]. The activation of reserve nutrient uptake of seed is evident. But these researches have a weakness. There are no any results which would show how the changes of radiative power and of the treatment durability value influence the researched parameter value.

Another factor plays an important role in the mechanism of ultrasound treatment of seeds and plants. The change of water properties and an intense influence of such water on biochemical processes in plants and seeds irrigated by such water are discovered by the authors. The capability of soil and other fine finders to preserve some properties of such water after the irrigation by such water is discovered. After full drying of such soil and the second irrigation by the main water the cavitated water properties permitting to influence biological objects are manifested again. I.e. the soil "memorizes" the properties of cavitated water and it reproduces them even after its full drying and its further irrigation by the main water.

The term "memory of soils" was mentioned earlier in connection with the following contexts:

- use of manure in the past. It reasons a higher yield in the next years and bates demands to $\mathrm{Ph}$ and $\mathrm{K}$ fertilizers [4]; 
- soil microorganisms, which are transferred from the plant to its descendants directly or through seedcase or through other structures [5];

- processes which took place in precedent soils and biosphere influence on the current soil [6];

- paleoecologic reconstruction, i.e. the soil evolution [7].

It is possible to add a new meaning of this term - the capability to memorize and then to reproduce the properties of water received in cavitation treatment. In addition, the solution based on cavitated water gets these additional properties.

\section{Materials and methods}

Vortex cavitator as the most multifunctional unit for sound processing of liquid medium was used [8].

The target of the research was seeds of various crops. So, in November of 2013 barley seeds were sown in the soil treated with the following procedure. At first it was irrigated by cavitated water, then it was completely dried and cut and after that the soil was irrigated by main-water. The following results in the germinating capacity (germinative energy) and in the development of plants were received [9].
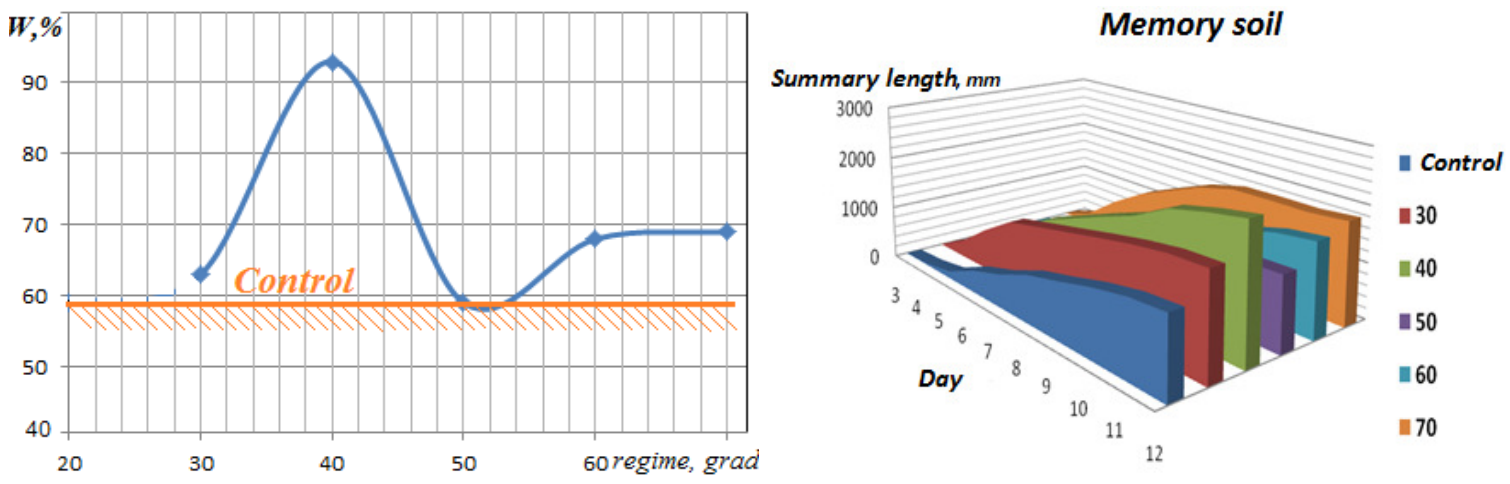

Fig. 1. Results in germinating capacity and in development of barley

The analysis of the results shows that the control plants had minimum germinating capacity and plant development rate during all the period of their germination and development. The maximum value in the germinating capacity and length average was achieved by the plants irrigated by the cavitated water received in the cavitator at a temperature of $40^{\circ}$ (Fig. 1).

The same regularities are proper to other crops (Fig. 2).

The differential studies in the following parameters were made: cavitated water irrigation; germination on napkins moistened by cavitated water, dried and moistened by main-water; and germination in the soil initially irrigated by cavitated water, then completely dried and after that irrigated by main-water.

The confidence intervals are determined in order to assess the individuality of each of the compared builds, in this case using the well-known technique for tripling $\mathrm{n}=3$

$$
X= \pm \frac{t_{\gamma}}{\sqrt{n}} \cdot S,
$$

at significance levels $\gamma=0.95\left(t_{\gamma}=0.315\right)$ of each of the compared relations by the method of sequential calculation of dispersion $D$, effective dispersion

$$
S^{2}=\frac{n}{n-1} D
$$

and the confidence interval $X(1)$. 
a)

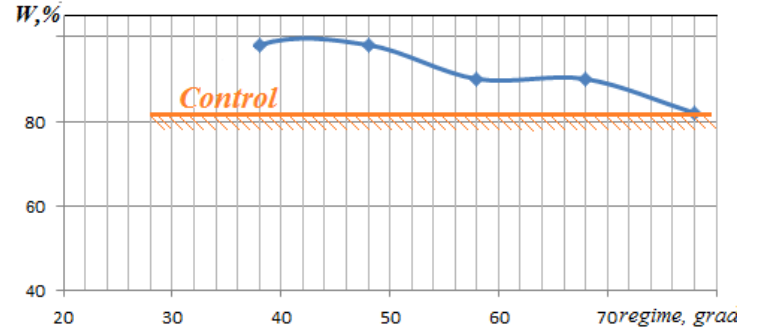

c)

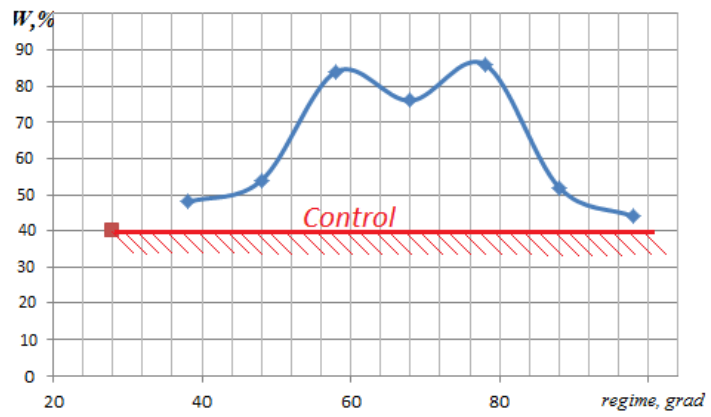

b)

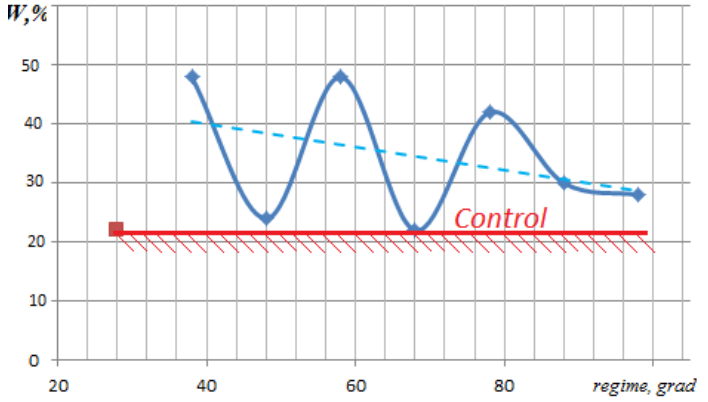

d)

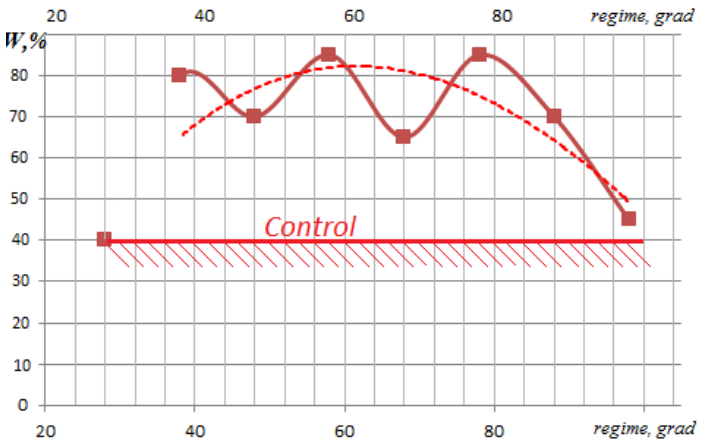

e)

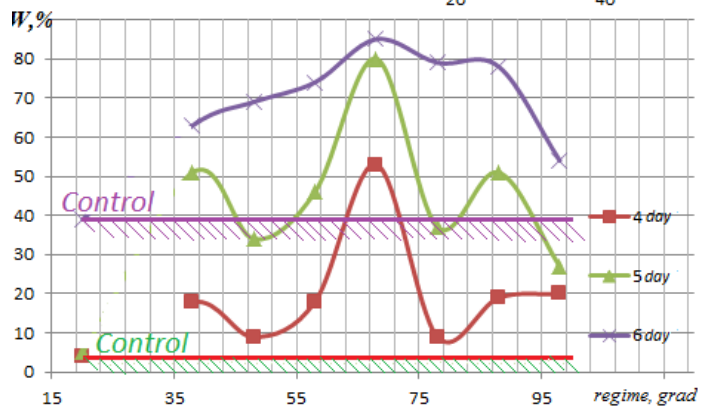

Fig. 2. Germinating capacity results of various crops:

$\mathrm{a}$ - tomato; $\mathrm{b}$ - pine; $\mathrm{c}$ - rye; $\mathrm{d}$ - wheat; $\mathrm{e}$ - sunflower

\section{Results and discussion}

It was discovered in previous researches that treatment of seeds in the cavitator as well as out of the cavitator by cavitated water induces changes of the seed germinating capacity and the further development of plants (Fig. 3). At that it was discovered that the dependence of the germinating capacity on any correction measure is cyclical [9].

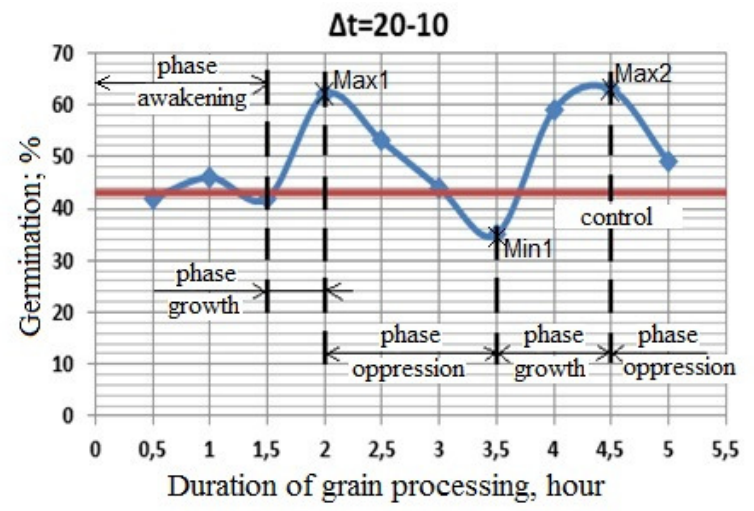

Fig. 3. Dependence of barley seed germinating capacity on duration of treatment $\Delta t=20-10^{\circ}$

These interleaved extremes denote the dependence periodicity and it permits to distinguish the following vital activity phases in the processes of their treatment in the cavitator: 
- awakening phase: the seeds have not yet awoken, but accumulation of the impact quantity (impact intensity $\times$ duration) takes place. The germinating capacity in $\Delta t=20-10^{\circ}$ is comparable with that of the control group moistened at the same temperature;

- growth phase: by its beginning $(1,5 \mathrm{~h})$ the impact quantity has achieved its level threshold and the seeds taken from the cavitator begin to develop and to germinate intensively (62\%);

- oppression phase: the seeds awoken in the previous phases would begin to develop and to germinate, but the conditions of their locating in the cavitator are not favorable for it. That is why each of the following group taken from the cavitator shows a decreased germinating capacity.

At that, in the oppression phase the immune system activates in each seed and it adapts the development process (after awakens) to the current conditions and it induces the further vital activity phase of seed in the conditions of their cavitation treatment:

- recurrent growth phase: at first, the germinating capacity of seeds increases up to $59 \%$ and, if the seeds are preserved in the cavitator a half an hour longer, it achieves $63 \%$. The growth rate in this phase is lower by exposure time but it is higher by quantitative measures than that of the first phase;

- further oppression phase. It is similar to the same phase of the first cycle, because, firstly, the germinating capacity decreases, and secondly, the awoken and ready to grow seeds are in conditions which are not favorable for growth. If the seeds are in such conditions for a short period of time, their growth is on the brake.

In the series of the experiments the seeds were watered in Petri dishes by water cavitated in different modes. The curve $\mathrm{W}=\mathrm{f}(\mathrm{t})$ (Fig. 4) characterizing this process is periodic like the abovementioned curve. But its trend line is increasing.

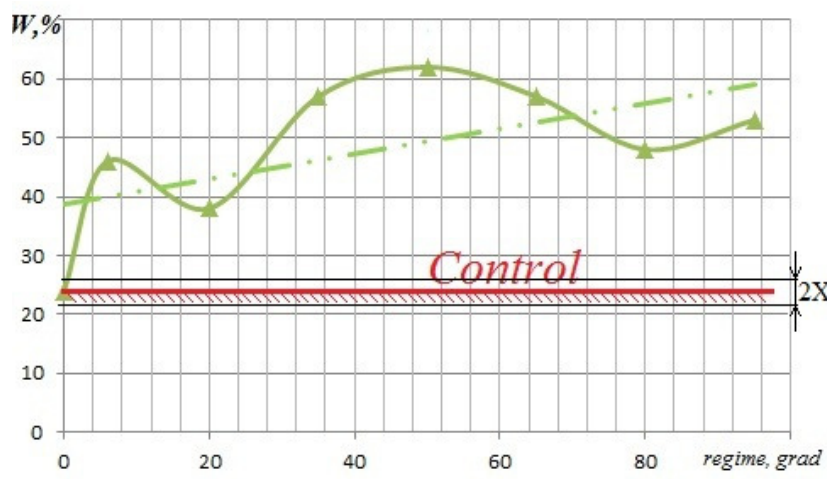

Fig. 4. Cyclical pattern of oat seed germinating capacity depending on different modes of water cavitation in Petri dish sprouting

This fact suggests that generally the germinating capacity is higher, if the treatment temperature is higher, because the bioactive substances appear. In this case the temperature mode indicates the number of cycles which particulates go in the circulation circuit of the cavitator.

$$
n=\frac{Q \cdot \tau}{V},
$$

where $Q$-pumping in the cavitator, $\mathrm{m}^{3} \cdot \mathrm{s}^{-1}$;

$V$ - volume of the internal cavity of the cavitator, $\mathrm{m}^{3}$;

$\tau$ - time of water exposition, $\mathrm{s}$.

The mode temperature is directly associated with the number of cycles of water movement in the circuit per unit time, because the appearance of bioactive substances is associated with the change in the structures of water clusters. The changes of the cluster structure are accompanied by the output (in this case) of their appearance in energy differences [10;11].

The process periodicity may be explained by the variable periodic quantity of generated, bioactive substances, but it is hardly probable, whether by the interaction nature of bioactive substances with the 
immune system of seeds. Firstly, the bioactive substances are external irritants by their nature and they induce waken seeds. Secondly, they stimulate the processes of the biological system development by increasing the cell membrane permeability, by synchronizing the function of certain subsystems. These facts are proper to the first increasing part of the function $W=f(t)$. But when the concentration of bioactive substances is higher, their influence on seeds becomes painful. On the other hand, in this case the bioactive substances influence only on a part of the biological system, provoking the dysfunction of the general system, which is comparable by its volume with the size of awoken elements in the initial increasing part of bioactive substances. The beginning of the next cycle (the increase of $W=f(t))$ acts with the further increase of the bioactive substance concentration. But the seeds are irritated on a higher energetic level: not some fragments awaken, but the vital activity systems are involved on a higher degree in the process. For that it is necessary to have more bioactive substances. The next phase of the second cycle has the same regularities than the analogical of the first cycle, but it acts on a higher energetic level.

However, if the temperature of water is near the atmospheric boiling point, i.e., $90-100^{\circ}$, the effectiveness of acoustic cavitation attack is considerably lower. So, distillated and boiled waters have the lowest biological activity, comparing all kinds of waters by their treatment and origin [12].

The germination in Petri dishes on napkins moistened by cavitated water, then dried and moistened by main-water, supposes the realization of the following mechanism of seed treatment.

Water besides ionic binding between molecules has hydrogen bindings, which induce the groupment of molecules in assemblies - clusters. These clusters may have various structures. Different configuration of molecules in a cluster, firstly, reasons different properties of water with clusters of different structures. Secondly, one form of molecule organization in clusters may be transformed to another. Usually it is accompanied by the energy release or consumption. It depends on the clustered formation energy value of any concret structure. Some of clusters are bioactive substances.

Another considerable factor of cluster existence is pollutants. Water is a very good solvent. Pollutants may represent finely dispersed particulate matters as well as dissolved salt ions, or fragments of other liquid, etc.

The minute particles of these pollutants entering inside each cluster touch water molecules creating a matrix corresponding to the cluster structure. Each matrix represents the memory of the previous cluster structure, i.e. it keeps the information, which may be reproduced, even if the water of this mixture will completely dry and water with another kind of clusters will be poured.

If this kind of seed soaking is chosen, the nap underlaying structure will contain some firm pulverescent particles. It may be fragments of villi as well as rubbish adsorbed from the outside and the particles of water. However, the quantity of such particles is not enough to create a matrix from the fragments watered with water having the required structure. That is why the germinating capacity in this variant is lower than in that of watering in cavitated water. If the temperature $t=50^{\circ}$, this difference achieves $\Delta W=20 \%$ (sector 2) (Fig. 5).

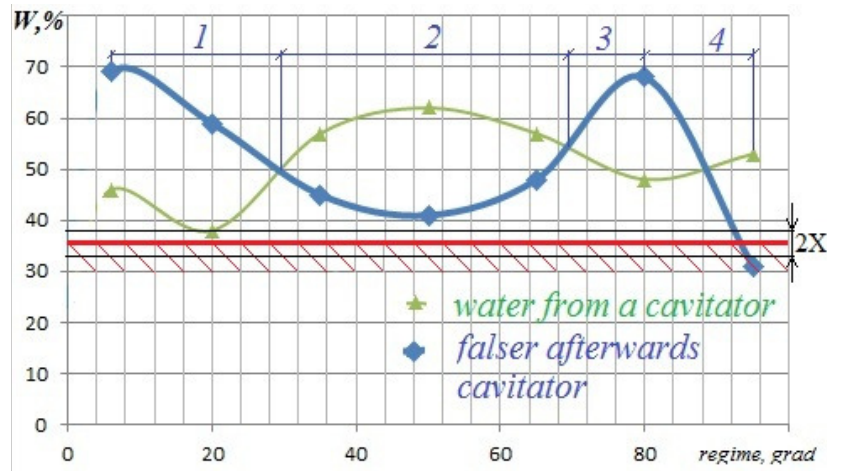

Fig. 5. Germinating capacity of oat seeds depending on water treatment mode in cavitator and in sprouting on fibrous layers in Petri dishes

There are some exceptions - the very beginning of the temperature mode $30^{\circ}>t>60^{\circ}$ (Sector 1). There another mechanism of bioactive substance formation is in action. So, the mode $t=6^{\circ}$ is a special mode of so called "polluted water" (the water streamed in the off-load cavitator and it was not treated). 
It suffers a more delicate treatment mechanism, which is associated with the formation and manifestation of magnetic, electrical, and other physical fields [13].

It the temperature is higher than $\mathrm{t}=50^{\circ}$, other factors influence. Firstly, the corrosive properties become stronger; secondly, the concentration of bioactive substances in the initial composition of water used for nap impregnation increases. In this case a higher number of bioactive substances structures will create more matrixes, in which a higher number of bioactive substances will be created after pouring main water. A higher number of such bioactive substances will be created in the final water after soaking. That is why the germinating capacity is higher in the modes with further increase of the circle number (or temperature) (sector 3 ).

The change of the dependency $W=f(t)$ in the sector 3 reflects the decrease in the germinating capacity, when the temperature becomes higher than $t=80^{\circ}$. It is associated with the approach to the atmospheric boiling point, in which the cluster structure changes by another mechanism and another structure, not favorable, appear. Consequently, each matrix appearing in these modes will create not favorable structures in the stage of drying and second watering [1]. The germinating capacity in the sector 4 decreases. If the temperature is more than $88^{\circ}$, the germinating capacity of such seeds is lower than treated directly in the cavitator. If the temperature is more than $88^{\circ}$, the structure becomes not favorable because of the lack of pollutants in use of clear nap layer.

If the seeds are sown in soil initially irrigated with cavitated water ( 7 variants), then dried and after that irrigated with main water, their germinating capacity is considerably higher than that of the control seeds. (Fig. 6). It proves once again the effect of the "memory of the soil" made by cavitated water.

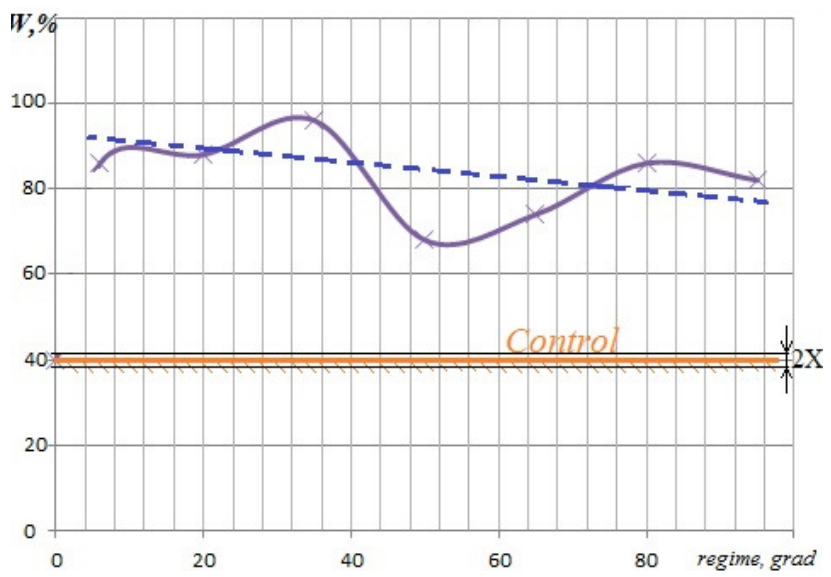

Fig. 6. Germinating capacity of oat seeds depending on modes of water treatment in cavitator in soil initially irrigated with cavitated water, then dried and after that irrigated with main water

At that, the trend of decrease in the germinating capacity $\mathrm{W}$, after the increase in liquid cycles in the cavitator (temperature level mode $t$ ), take places. If the number of cavitation impact on the initial water increases, the number of bioactive substances increases, too. As there are many polluants in the soil, they transfer in the water using for irrigation. The overnumber of bioactive substances in the irrigation water depresses the seeds. Besides, there is a cyclicity of the germinating capacity dependency $W=f(t)$ : first cycle $t=60 \div t=50^{\circ}$, second cycle $t=50 \div t=95^{\circ}$. The nature of cyclicity in this case is the same as that, if the seeds are moistened directly in cavitated water, but with an additional fact: the presence of any additional chemical elements and substances. That is why in this case the displacement of homonymous extremes relative to $t$ modes takes place. So, in the case of cavitated water $\mathrm{t}=50^{\circ}$ there is maximum in the germinating capacity W. In the case of germination in soil initially irrigated with cavitated water, then dried and after that irrigated with main water in $t=50^{\circ}$, there is minimum in the germinating capacity $\mathrm{W}$. This phenomenon also manifests in the growth stage. This mode is the most unfavorable for planting in soil for this crop (oats).

Besides, there is once more unfavorable mode by the plant average length criterion in the growth stage in $t=80^{\circ}$ (Fig. 7). 


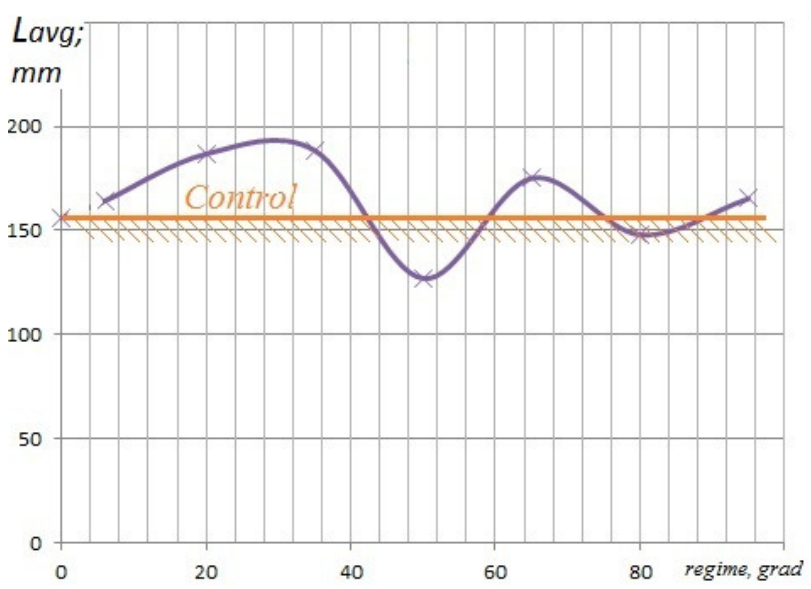

Fig. 7. Intensity of plant growth from oat seeds depending on modes of water treatment in cavitator in germination in soil taking into account the "soil memory"

However, by the Caulis total length criterion (green masse in the experiment) all kinds of water treatment override the control variant (Fig. 8).

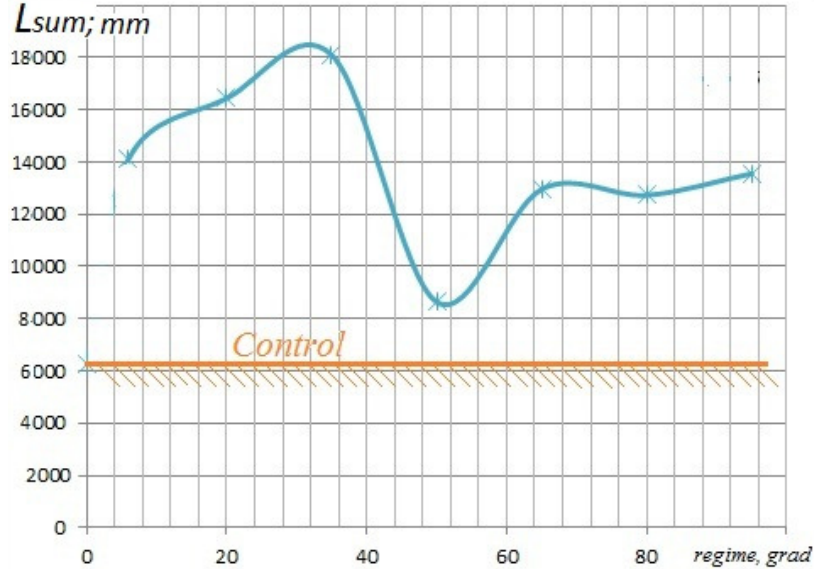

Fig. 8. Integral estimation of intensity of plant growth from oat seeds depending on modes of water treatment in cavitator in germination in soil taking into account the "soil memory"

The above-mentioned research results are proved in the series of experiments for influence of the "soil memory" on sunflower seeds. Here the irrigation with cavitated water is more effective than the control and the results with the "soil memory" are higher by the germinating capacity and plant length only than that of the irrigation with treated water (Fig. 9).

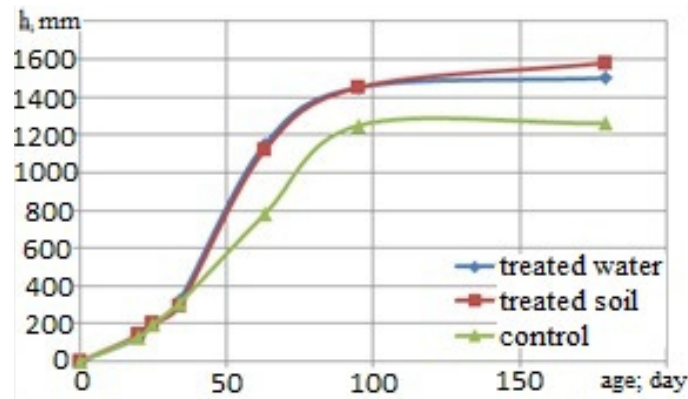

Fig. 9. Regularities of sunflower plant growth in different stimulation modes:

seeds are moistened in cavitated water; planted in soil which had been irrigated several months ago with cavitated water, then dried; control party

The received regularities prove the reality of the "soil memory" effect as a result of its irrigation with cavitated water. This phenomenon permits not only to increase a positive influence on the growth and development of various crops, but also to economize the water resource by keeping and transmission of its properties through soil. 


\section{Conclusions}

1. The information capacities of water are discovered (proved one more time) by use of biological indicators. They are manifested in different seed germination capacities and different intensities of development of plants irrigated by cavitated water in different modes.

2. It is evident that it is possible to determine the wanted capabilities of water by creation of bioactive substances by the change of the water cluster structure by cavitation.

3. The hydrodynamic units for acoustic cavitating treatment of water are created, manufactured, and approbated.

4. It is established that it is possible to conserve and then to transfer the information which the water received from the cavitator after the soak of fine finders for examples of soil. Even if the soil is completely dried, these properties are reproduced again after irrigation with main water. It is proved by the germination capacity tests of barley, tomato, pine, rye, wheat, and sunflower.

5. The percentage of information conserved in fine finders is reasoned by the dispersity index and the diversity of the material composition. The germination capacity tests showed that the soil conserved more properties which water received from the cavitator than homogeneous fibrous material.

6. It is discovered that this way may be used in order to economize clear fresh water by frequent use of "bad water" for irrigation after seldom preliminary irrigation by "good water".

7. This paper is initial in the study of information properties of water. It is of current importance for solving applied tasks and for the solving of astrophysical problems, because the most part of the planet surface is occupied by water.

\section{References}

[1] Hmeliova A.N. The influence of ultrasonic irradiation on rhizogenous activity of plant objects in combination with growth regulators: authors abstract Ph.D (biology) 03.00.16. Hmeliova Anna Nikolaevna, Barnaul, 2009. 21 p.

[2] Tarasov S.S. Catalase activity in the seeds of cereals in normal and under the influence of ultrasound. Proceedings of all-Russian Council of young scientists and specialists of agricultural educational and scientific institutions "Current problems and mechanisms of development of agriculture", 2018, pp. 20-24.

[3] Tarasov S.S., Veselov A.P. Ultrasound effect on the morphological and physiological indicators of seed germination of pea (Pisumsativum L.). News of higher educational institutions. Volga region. Natural science, 2018, No 3.

[4] Njroge S., Schut G.T., Giller K.E. etc. Learning from the soil's memory: Tailoring of fertilizer application based on past manure applications increases fertilizer use efficiency and crop productivity on Kenyan smallholder farms. European Journal of Agronomi, vol. 105, 2019, pp. 52-61.

[5] Lapsansky E.R., Milroy A.M., Andales M.J. etc. Soil memory as a potential mechanism for encouraging sustainable plant health and productivity. Current Opinion Biotechnology, vol. 38, 2016, pp. 137-142.

[6] Yaalon D. Comments on Soil Memory and Paleo-Environmental Reconstruction of Soil Evolution. Eurasian Soil Science, vol. 44, 2011, No 4, pp. 505-506.

[7] Targulian V.O., Goryachkin S.V. Soil Memory and Environmental Rekonstructions. Evrasian Soil Science, vol. 44, 2014, No 4, pp. 464-465.

[8] Ivanov E.G. Evolution of hydrodynamic cavitator. Mechanization in Agriculture, issue 2, 2015, pp. 17-21.

[9] Agafonov V.V., Ivanov E.G., Chavachina E.E. Cavitation germination of barley seeds for accelerated malt manufacturing. Bulletin of NNSEEI, 2016, No 8(63), p. 55-64.

[10]Zenin S.V., Tyaglov B.V. Hydrophobic model of associate structur of water molecules. Russian Journal of Physical Chemistry, vol. 68, 1994, No 4, pp. 636-641.

[11]Zaharov S.D., Mosyagina I.V. Kluster structure of water (survey). Moscow: Lebedev Physical Institute RSA, 2011. 24 p.

[12]Zalepuhin V.D., Zalepuhin I.D. Key to "life-giving water". Alma-Ata: Kainar, 1987.

[13] Ivanov E.G. Seed germination with use of acoustic-cavitation tehnologies. Proceedings of 15th International scientific conference "Engineering for rural development", vol. 5, May 25-27, 2016, Jelgava, Latvia, pp. 278-284. 